\title{
COMPORTAMIENTO DEL CONSUMIDOR DE PRODUCTOS EN LA OFERTA DE TRIBU INGA
}

\section{BEHAVIOR OF THE CONSUMER OF PRODUCTS IN THE OFFER OF INDIGENOUS INGA TRIBES}

\author{
Mawency Vergel-Ortega ${ }^{1}$, Luisa Stella Paz-Montes ${ }^{2}$ y Jhan Piero Rojas-Suárez ${ }^{3}$ \\ ${ }^{1}$ Universidad Francisco de Paula Santander, Colombia. Email: mawency@ufps.edu.co \\ ${ }^{2}$ Universidad Francisco de Paula Santander, Colombia.Email: luisastellapm@ufps.edu.co \\ ${ }^{3}$ Universidad Francisco de Paula Santander, Colombia. Email: jhanpierorojas@ufps.edu.co
}

Para citar este artículo: Vergel, O. M., Paz-Montes, S. L. y Suárez, R. J. (2019). Comportamiento del consumidor de productos en la oferta de tribus indígenas. Clío América, 13(25), 276-286. doi: http://dx.doi.org/10.21676/23897848.3408

Recibido: 06 marzo de 2019

Aceptado: 20 de mayo de 2019

Publicado en línea: mayo 24 de 2019

\section{Palabras clave:}

Tradición;

economía;

marketing;

tribus; inga.

JEL: Z1; Z3.

\section{RESUMEN}

La investigación sigue un enfoque mixto. Su objetivo es diseñar un modelo estructural que identifique variables explicativas del proceso de compra de artesanías y extractos de plantas medicinales a la comunidad inga. Desde el método etnográfico en investigación cualitativa, y utilizando elementos del diseño factorial y correlacional, basado en el modelo de ecuaciones estructurales (SEM) del enfoque cuantitativo, se implementan instrumentos de entrevista, observación y encuesta a una muestra constituida por 500 transeúntes consumidores de productos naturales y 280 integrantes de la comunidad inga del municipio de Cúcuta, Colombia. Los resultados permiten identificar categorías emergentes como planeación territorial participativa, contexto, igualdad de derechos, producción sustentable, organización, investigación e innovación, mercadeo y capacitaciones como inversión natural; así mismo, dentro de una inversión ambiental, factores de capacidad de talento humano, uso de prácticas ancestrales, factores sociales, producto, afecto y tecnología inciden en el comportamiento del consumidor como irrupción del orden social. Se concluye que a partir del modelo logit se identifica la representación cultural influida por el comportamiento del consumidor de productos en la oferta de las tribus.

\section{ABSTRACT}

The research follows a mixed approach; and aims to design a structural model that identifies explanatory variables in the process of purchasing crafts and medicinal plant extracts from the Inga community. Using the ethnographic method in qualitative research and using elements of the factorial and correlational design, based on the structural equation model (SEM) of the quantitative approach, instruments are implemented for interviewing, observing and surveying a sample made up of 500 passers-by who consume natural products and 280 members of the Inga community in the municipality of CúcutaColombia. The results allow us to identify emerging categories such as participatory territorial planning, context, equality of rights, sustainable production, organization, research and innovation, marketing, training as a natural investment, as well as, within an environmental investment, factors of human talent capacity, use of ancestral practices, social factors, product, affection, technology; all of which affect consumer behavior as an irruption of the social order. It is concluded that, from the logit model, the cultural representation influenced by the consumer's behavior of products in the offer of the tribes is identified. 


\section{COMPORTAMIENTO DEL CONSUMIDOR DE PRODUCTOS EN LA OFERTA DE TRIBU INGA}

\section{INTRODUCCIÓN}

La economía y la cultura empresarial y sectorial permiten analizar las relaciones que hay en el proceso de toma de decisiones, asociado al comportamiento del consumidor, que involucra recursos, actores como comprador, adquisición y el bien o servicio recibido (Vilela, Vergel y Paz, 2018). El análisis de los impactos económicos en la población, en diferentes ámbitos, ya sean locales, globales o de políticas culturales, se constituye en insumo útil para analizar y valorar inversiones realizadas, así como para evaluar los diferentes programas que implementan entidades o colectivos y sus nuevas estrategias en el área pública, privada o empresarial. Para este fin, la investigación buscó identificar representaciones culturales del mercadeo en la tribu inga, indígenas cuyo plan de salvaguarda señala que se localizan en zona rural de los departamentos Norte de Santander, Arauca, Putumayo, Cauca, Caquetá y Nariño. Esta tribu, por diferentes circunstancias, se ha desplazado hacia el Norte de Santander, y ha habitado en ciudades como Cúcuta.

En cuanto a las investigaciones asociadas a los temas relacionados con las culturas indígenas, se destaca el trabajo de Duarte, Ortega, Mora y Coromoto (2011). Estos autores buscaron establecer un modelo de salud basado en la medicina ancestral del pueblo wayuu. Su investigación señala un perfil epidemiológico caracterizado por enfermedades y pobreza, asociado a la ubicación geográfica (escasez de agua). La tribu inga, establecida en Cúcuta, se conforma por aproximadamente 280 personas, distribuidas de 73 a 90 familias dispersas en estos barrios: Antonia Santos, Los Olivos, San Martín, Los Alpes y Santa Teresita, Bogotá, 28 de Febrero, Ceci, Belisario, Aguas Calientes, Belén, Toledo Plata, Las Coralinas, La Unión, Nueva Esperanza, Sabana Verde, San Miguel, Santo Domingo, Camilo Torres, Gaitán y Buenos Aires.

Desde épocas pasadas, los integrantes de las tribus indígenas vienen cursando programas académicos universitarios, y en ese contexto han llevado conocimiento a los integrantes de las tribus. Saberes como la matemática y su aplicación han permeado las culturas al implementar constructos administrativos; han llevado la ciencia a la vida cotidiana para generar y proyectar sus creencias en la producción de plantas medicinales, que sirvan para el beneficio. Además, han sido conocimientos útiles para la fabricación y distribución de medicamentos. Esto también ha influido en la distribución de tierras de cultivo, donde se prevé usar la gestión empresarial. Sin embargo, no existen estudios orientados a esta población que permitan analizar el comportamiento del consumidor frente a los productos que ofrecen las comunidades indígenas.
En la tribu inga consideran que la medicina y el ritual deben realizarse por territorio; cada individuo actúa según su condición física y su realidad espiritual, y acorde con el lugar donde nació. Estas características las asocian a símbolos, figuras geométricas, al intercambio de bienes, pero una gran parte de la población desconoce su origen, significado o utilidad, y solo lo implementan por tradición de sus ancestros. Sin acudir en ninguna instancia, resuelven cualquier problema, desde transacciones, distribución de terrenos, cultivo eficiente, cura de infecciones, venta de artesanías, plantas o brebajes hasta la atención de partos.

Por lo anterior, es muy importante tener claridad sobre el pensamiento económico de las tribus, el manejo de los conceptos empíricos que les permiten realizar transacciones, así como su aplicación en el cultivo, la producción, el uso y la distribución de plantas medicinales. Con base en esto, es necesario brindar una orientación académica, integrar currículos y aprovechar conocimientos y creencias para generar nuevo conocimiento comprobado científicamente. De acuerdo con lo anterior, la investigación responde a la pregunta: ¿Qué modelo predictivo permite analizar el comportamiento del consumidor en la oferta o el mercadeo de la tribu inga?

\section{METODOLOGÍA}

El enfoque metodológico mixto, basado inicialmente en el cualitativo de tipo etnográfico (Guber, 2011), descriptivo, prospectivo y analítico, permitió trabajar la realidad del objetivo de la investigación (Vergel, Martínez y Nieto, 2016), además de sus costumbres y actitudes (Rojas, Vergel y Urbina, 2019). El tamaño de la muestra estuvo conformado por 500 informantes claves de la tribu inga de Cúcuta, transeúntes del sector centro y de las zonas habitables por los ingas. Para la selección de la muestra se tuvo en cuenta el muestreo por bola de nieve o cadena (López, 2004), en la que el primer informante da su aporte cultural y, posteriormente, indica a otra persona que consume o ha adquirido productos y cuidados culturales especiales, hasta lograr la saturación óptima para analizar insumos.

Respecto al método cualitativo, la etnografía tiene su objeto de estudio, según Murillo (2010), en la comprensión del ser humano, vista y analizada como un proceso, con una concepción holística (Spradley, 2008) en la que se pueden estudiar los diferentes componentes culturales (Hevia, 2002). En este caso, el estudio se centra en las representaciones culturales de la tribu inga, frente a sus diferentes actuaciones, mediante sus creencias y sabiduría. En cuanto al enfoque cuantitativo, este sigue un método correlacional explicativo. $\mathrm{Al}$ asumir los procedimientos de 
Martínez, Vergel y Zafra (2016), que parten de lo cualitativo y llevan a analizar frecuencias que luego, a través de modelos, permiten el análisis de las probabilidades de compra condicionadas al estado emocional del individuo, la investigación constó de tres fases. La primera de estas, de carácter descriptivo, analizó la satisfacción del uso del producto medicinal o artesanal por temporada; a partir de series de tiempo se buscan modelos asociados a variables (Rueda, Romero y Gómez, 2019), como compra, consumo, satisfacción y variables socioeconómicas y demográficas que caracterizan al individuo, la segunda fase implica el análisis de información frente al modelo y la tercer fase análisis de correlaciones y validez del modelo.

El modelo propuesto, al asumir algunas variables y preceptos de Marcillo y Zambrano (2010), considera variables como 1$)$ género $(\mathrm{G} 1$, donde 1 es mujer, 0 para otro), 2) edad en años, 3) edad al cuadrado, 4) educación primaria, 5) educación secundaria, 6) educación superior, 7) nivel de ingreso, 8) nivel de posgrado, 9) categorías asociadas a la medicina natural en torno a las creencias, 10) medicina alternativa, 11) venta de artesanía, 12) ritos, 13) asistencia, 14) estado emocional anterior. Las probabilidades para modelar se designan por:

$$
\pi_{01}(\mathrm{t})=\mathrm{P}\left(\mathrm{Y}_{\mathrm{tv}}=1 / \mathrm{Y}_{(\mathrm{t}-1)}=0\right) ; \pi_{11}(\mathrm{t})=\mathrm{P}\left(\mathrm{Y}_{\mathrm{t}}=1 / \mathrm{Y}_{(\mathrm{t}-1)}=1\right)
$$

Cada una de ellas corresponde a la tasa de adquisición de productos. Desde que solo se consideran individuos de la población económicamente activa resulta:

$$
\mathrm{P}\left(\mathrm{Y}_{\mathrm{t}}=1 / \mathrm{Y}_{(\mathrm{t}-1)}\right)+\mathrm{P}\left(\mathrm{Y}_{\mathrm{t}}=0 / \mathrm{Y}_{(\mathrm{t}-1)}\right)=1
$$

Se utiliza modelo tipo logit con explicativas $\{\mathrm{Xhi}(\mathrm{t}-1), \mathrm{h}=1$, ..., $\mathrm{H}, \mathrm{t}=2, \ldots, \mathrm{T}\}$ socioeconómicas y de carácter de ubicación. Se describen influencias sobre la tasa de compra, así como el hecho de haber adquirido productos o no en el periodo previo, a partir de un modelo en tiempo discreto. Los tiempos de observación incluyen respuestas de marzo a junio. Al seguir modelo de Agresti, según García (2012):

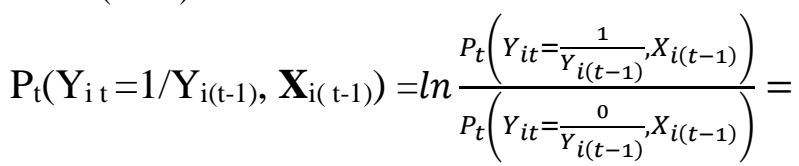

$$
\begin{aligned}
& \beta_{0 t}+\alpha_{(t-1)} Y_{i(t-1)}+\sum_{h=1}^{H} \beta_{h t} X_{h i(t-1)}
\end{aligned}
$$

En el periodo (t-1), variables en el vector $\mathrm{Xi}(\mathrm{t}-1), \pi 11(\mathrm{t})$ están relacionadas con la duración de compra.

\section{Cuestiones introductorias a representaciones culturales y sociales}

En el horizonte teórico, las representaciones culturales han sido influenciadas por el exterior a las tribus (Salaverry, 2010). Han venido siendo parte de la sociedad en común, conformadas por grupos que se diferencian por sus costumbres, tradiciones y creencias que identifican a una comunidad específica (Vergel, Delgado y Diaz, 2019), donde plasman su lenguaje, sus relaciones sociales y los ritos propios de cada grupo social. Todo esto se puede manifestar a partir del patrimonio cultural y de la identidad que representa. Según Jaramillo (1984), la representación cultural se define con base en la realidad ontológica y en su historia en el dialecto y el idioma, instrumentos con los cuales el colectivo comunica las ceremonias y ritos, elementos que le otorgan identidad cultural. De este modo, para cada integrante de dicha cultura, su grupo se constituye en un refugio para acudir ante cualquier acontecimiento de la vida, tanto en momentos de armonía como en situaciones difíciles.

Por su parte, López (2011) centra las representaciones sociales en los patrones de pensamiento social y comportamiento propios de la rutina cotidiana en el contexto concreto, que se relacionan con la manera como los sujetos afrontan su vida diaria y la relación con los demás (Jovchelovit, 2001), en un conocimiento que es socialmente elaborado y compartido (Sarrazin, 2015). Por otra parte, los modelos matemáticos asociados a la producción de plantas medicinales de las tribus inga, así como el uso de estas en los indígenas, están ligados a sus creencias milenarias, a su saber ancestral, a la complejidad del mundo de su vida, pues se transmiten en el proceso socializador, pero sufren transformaciones como realidades socialmente construidas, y en esa medida signadas por la subjetividad. El consumo de los productos determina la manera cómo afrontan el proceso de adquisición, según el canal de distribución. La representación de los indígenas acerca de la salud, la economía y la producción de las plantas se refiere a los principios de armonía entre la naturaleza (Duarte, et al., 2011), el cuerpo y su espíritu.

\section{RESULTADOS}

\section{Concepciones y percepciones en mercadeo}

El mercadeo para los integrantes de la tribu y para transeúntes se asocia al concepto de salud y tradición, que se relaciona con la opción de trabajar; representa tranquilidad y sociedad. El dinero lo perciben como un medio vinculado al placer, al poder y, algunos, al castigo, $\mathrm{y}$ afecta lo físico y lo emocional en cada uno; les impide 
actuar con normalidad en todas sus actividades, lo que perjudica no solo su bienestar, sino también su economía. Al evidenciar las conductas culturales utilizadas por la tribu inga para el mercadeo de sus productos, todos los miembros de la tribu conocen los modos de preparación, conservación y administración de medicamentos naturales como purgantes, además de secretos que no se transmiten a ningún miembro que no pertenezca a la tribu. Por eso se consideran un grupo social con conocimiento que les otorga dominio de actividades que pueden ayudar conseguir las metas que se proponen en equipo, anticipándose a los deseos de consumidores, y a desarrollar productos o servicios aptos para un mercado que los prefiere por ser una población vulnerable. Las definiciones dadas por la tribu y la teoría coinciden significativamente por cuanto los principios del marketing, sean emocionales, psicológicos y espirituales, son expresados por la tribu.

\section{Representaciones sociales: proceso y prácticas}

Frente al proceso y las prácticas de microdesarrollo regional, surgen las categorías emergentes planeación territorial, contexto, equidad de género, producción sustentable, investigación, mercadeo y capacitaciones (tabla 1). Las creencias giran alrededor de las tradiciones, la vida, la naturaleza y la salud. El establecer sitios sagrados señala rupturas de uso del espacio habitacional y urbano, donde muestran su comunicación con otros espacios y la conexión mundo-tierra-astronomíadimensiones espirituales. De esta manera, cada escenario en la tierra, cada objeto con vida - como el árbol, los animales, el agua - o inerte - como una roca, una ruta, una vía-, contiene significados que se convierten en símbolo, y propician un nuevo lenguaje y expresión de contacto con su mundo espiritual. Establecen una relación entre el lenguaje espacial y la realidad del lugar. Las categorías emergentes fueron dinero - subcategorías placer, poder, limpieza, yagé-, conductas culturales - subcategorías mercadeo, sitio sagrado, modo de preparación, administración, medicamentos secretos no transmitidos, dominio, actividad, consecución de metas, deseo de consumidores, desarrollo de productos - y tribu subcategoría marketing emocional, psicológico y espiritual一.

Tabla 1. Representaciones sociales: proceso y prácticas de microdesarrollo regional en la zona urbana

\begin{tabular}{|c|c|c|}
\hline Categoría & Objetivo & Concepciones asociadas \\
\hline \multirow[b]{2}{*}{$\begin{array}{l}\text { Planeación } \\
\text { territorial } \\
\text { participativa }\end{array}$} & Estrategias de desarrollo & \multirow{2}{*}{$\begin{array}{l}\text { Proyectos de producción individual, estratégicos, } \\
\text { e institucionales, tales como financiamiento, } \\
\text { certificación, capacitación, asesoría y promoción } \\
\text { de diversos tipos de producción agrícola. } \\
\text { Espacio y área de acción }\end{array}$} \\
\hline & $\begin{array}{l}\text { Participación y representatividad de los } \\
\text { indígenas para proponer y definir } \\
\text { procesos de desarrollo sustentable. }\end{array}$ & \\
\hline Contexto & $\begin{array}{l}\begin{array}{l}\text { Establecer el campo de ubicación, } \\
\text { dominio y acción territorial. }\end{array} \\
\end{array}$ & Espacio y área de acción \\
\hline $\begin{array}{l}\text { Igualdad } \\
\text { derechos }\end{array}$ & Permitir poder en la mujer. & $\begin{array}{l}\text { La mujer como representante de la tribu en la } \\
\text { ciudad para fomentar el diálogo con otras culturas. }\end{array}$ \\
\hline $\begin{array}{l}\text { Producción } \\
\text { sustentable }\end{array}$ & $\begin{array}{l}\text { El } 60 \% \text { de familias en proyectos } \\
\text { apoyados por la gobernación mejoran } \\
\text { sus ingresos y aumenta compra externa. }\end{array}$ & $\begin{array}{l}\text { Aprovechamiento sustentable en los recursos } \\
\text { naturales }\end{array}$ \\
\hline zación & $\begin{array}{l}\text { Nuevas actividades de entre gobierno } \\
\text { cabildo. }\end{array}$ & le planeación \\
\hline $\begin{array}{l}\text { Investigación e } \\
\text { innovación }\end{array}$ & $\begin{array}{l}\text { Ausencia de capacitación y proyección a } \\
\text { la comunidad en producción y } \\
\text { distribución de productos. }\end{array}$ & $\begin{array}{l}\text { Manifiesto deseo de promocionar sus productos e } \\
\text { innovar }\end{array}$ \\
\hline \multirow{4}{*}{ Mercadeo } & Visualización y comunicación & Trajes $\mathrm{t}$ \\
\hline & Salud & Manifiesto de vida \\
\hline & Tradición & Transmisión de conocimiento \\
\hline & Trabajo & $\begin{array}{l}\text { Actividad económica para gozar de otros } \\
\text { privilegios realizada en gran parte por el hombre }\end{array}$ \\
\hline \multirow{3}{*}{ Capacitaciones } & Tranquilidad & Paz, sintonía con la naturaleza \\
\hline & Sociedad & Culturas que rodean y acompañan \\
\hline & Capacitación y trabajo sistemático & $\begin{array}{l}\text { Enseñanza necesidad de progreso y difusión de } \\
\text { tradiciones }\end{array}$ \\
\hline
\end{tabular}

Fuente: elaboración propia basada en los datos obtenidos de entrevistas y protocolos de observación (2018) 
Si bien lo utilizan y algunos integrantes han convertido el dinero en un fin, un alto porcentaje lo ve como medio para obtener cosas materiales del mundo terrenal, lo que genera placer y poder. Se considera un castigo porque absorbe, y quien lo obtiene quiere, según ellos, conseguir más, lo cual los aleja de la vida espiritual. Semanalmente realizan ritos con yagé para limpiarse de las impurezas que el mundo externo les lleva. Sus conductas culturales se asocian a la venta y la producción, en especial de plantas medicinales, manillas y bolsos. Sus representaciones inciden de manera colectiva en todo el cabildo. Permean su funcionamiento cognitivo. Su mundo gira alrededor de la salud, la medicina, el conocimiento ancestral y su transmisión para la producción de extractos medicinales; está permeado por el contexto del mundo material que busca satisfacer consumidores y hacer más atractivo el producto a los ojos del consumidor, incluyendo en sus creencias la necesidad de realizar prácticas de mercadeo que permitan la venta y potencie sus tradiciones en la comunidad externa.

\section{Factores asociados a la zona rural}

Factores geográficos, sociales y de direccionamiento estratégico señalan la aparición de elementos de planificación y emprendimiento, con estructura organizativa, normativa, social y cultural que limitan sus fronteras, cuyo principio fundamental rige al pueblo inga en la ley primera (ancestral), la cual incide en la organización del espacio y el territorio (tabla 2).

Tabla 2. Productos y factores asociados zona rural

\begin{tabular}{|c|c|c|}
\hline Categoría & Actividad & Factor asociado amenaza \\
\hline Actividades agrícolas & $\begin{array}{l}\text { Siembra, recolección, beneficio y } \\
\text { secado en sus casas o fincas } \\
\text { Siembra y extracción de aceites en } \\
\text { casas }\end{array}$ & $\begin{array}{l}\text { Café pergamino seco } \\
\text { Preferencia planta medicinal supermercado } \\
\text { Duración plantas } \\
\text { Reconocimiento de marcas }\end{array}$ \\
\hline Actividades agrícolas & $\begin{array}{l}\text { Concentrados } \\
\text { Extracto medicinal }\end{array}$ & \\
\hline \multirow[t]{2}{*}{ Producción } & Área de cultivo & $54 \% 1$ hectárea \\
\hline & $\begin{array}{l}\text { Comunidad } \\
\text { minifundista }\end{array}$ & Con cultivos inferiores a 1000 matas \\
\hline $\begin{array}{l}\text { Direccionamiento } \\
\text { estratégico }\end{array}$ & $\begin{array}{l}\text { Gobernador del resguardo y los } \\
\text { integrantes del Cabildo Menor de } \\
\text { Economía }\end{array}$ & Ambiente externo \\
\hline Factores tecnológicos & $\begin{array}{l}\text { La amenaza mayor es la } \\
\text { utilización de tecnología de punta } \\
\text { por parte de la competencia }\end{array}$ & $\begin{array}{l}\text { El resguardo opera con técnicas agrícolas } \\
\text { ancestrales, haciendo que sus procesos sean } \\
\text { más lentos frente a su competencia. }\end{array}$ \\
\hline Factores económicos & Productos & Energizantes, homeópatas, remedios caseros \\
\hline \multirow[t]{2}{*}{ Factores competitivos } & Prácticas agrícolas y de mercadeo & $\begin{array}{l}\text { Plagas } \\
\text { No marca } \\
\text { No reconocidos por Invima } \\
\text { No acceso a mercados } \\
\text { y aumentar sus ventas }\end{array}$ \\
\hline & Proceso productivo & Tecnología \\
\hline Factores geográficos: & Clima, suelo & $\begin{array}{l}\text { Por sus identidades étnicas y culturales son } \\
\text { consideradas poblaciones uniformes y } \\
\text { legalmente reconocidas, facilitando la } \\
\text { participación como comunidad. }\end{array}$ \\
\hline Factores sociales & $\begin{array}{l}\text { Creencias } \\
\text { Buenas prácticas saludables }\end{array}$ & $\begin{array}{l}\text { El conocimiento de la sociedad no permite hoy } \\
\text { día la adquisición de productos que no cuentan } \\
\text { con registros de entidades gubernamentales de } \\
\text { salud a fin de garantizar la seguridad de la } \\
\text { salud. }\end{array}$ \\
\hline Categoría & Actividad & Factor asociado amenaza \\
\hline Actividades agrícolas & $\begin{array}{l}\text { Siembra, recolección, beneficio y } \\
\text { secado en sus casas o fincas }\end{array}$ & $\begin{array}{l}\text { Café pergamino seco } \\
\text { Preferencia planta medicinal supermercado }\end{array}$ \\
\hline
\end{tabular}


COMPORTAMIENTO DEL CONSUMIDOR DE PRODUCTOS EN LA OFERTA DE TRIBU INGA

\begin{tabular}{|c|c|c|}
\hline & $\begin{array}{l}\text { Siembra y extracción de aceites en } \\
\text { casas }\end{array}$ & $\begin{array}{l}\text { Duración plantas } \\
\text { Reconocimiento de marcas }\end{array}$ \\
\hline $\begin{array}{l}\text { Proceso y producto } \\
\text { final }\end{array}$ & $\begin{array}{l}\text { Concentrados } \\
\text { Extracto medicinal }\end{array}$ & \\
\hline \multirow[t]{2}{*}{ Producción } & Área de cultivo & $54 \% 1$ hectárea \\
\hline & $\begin{array}{l}\text { Comunidad } \\
\text { minifundista }\end{array}$ & Con cultivos inferiores a 1000 matas \\
\hline $\begin{array}{l}\text { Direccionamiento } \\
\text { estratégico }\end{array}$ & $\begin{array}{l}\text { Gobernador del resguardo y los } \\
\text { integrantes del Cabildo Menor de } \\
\text { Economía }\end{array}$ & Ambiente externo \\
\hline Factores tecnológicos & $\begin{array}{l}\text { La amenaza mayor es la } \\
\text { utilización de tecnología de punta } \\
\text { por parte de la competencia }\end{array}$ & $\begin{array}{l}\text { El resguardo opera con técnicas agrícolas } \\
\text { ancestrales, haciendo que sus procesos sean } \\
\text { más lentos frente a su competencia. }\end{array}$ \\
\hline Factores económicos & Productos & Energizantes, homeópatas, remedios caseros \\
\hline \multirow[t]{2}{*}{ Factores competitivos } & Prácticas agrícolas y de mercadeo & $\begin{array}{l}\text { Plagas } \\
\text { No marca } \\
\text { No reconocidos por Invima } \\
\text { No acceso a mercados } \\
\text { y aumentar sus ventas }\end{array}$ \\
\hline & Proceso productivo & Tecnología \\
\hline Factores geográficos: & Clima, suelo & $\begin{array}{l}\text { Por sus identidades étnicas y culturales son } \\
\text { consideradas poblaciones uniformes y } \\
\text { legalmente reconocidas, facilitando la } \\
\text { participación como comunidad. }\end{array}$ \\
\hline Factores sociales & $\begin{array}{l}\text { Creencias } \\
\text { Buenas prácticas saludables }\end{array}$ & $\begin{array}{l}\text { El conocimiento de la sociedad no permite hoy } \\
\text { día la adquisición de productos que no cuentan } \\
\text { con registros de entidades gubernamentales de } \\
\text { salud a fin de garantizar la seguridad de la } \\
\text { salud. }\end{array}$ \\
\hline
\end{tabular}

Fuente: elaboración propia basada en los datos obtenidos de entrevistas y protocolos de observación (2018).

Por otra parte, el análisis de ambiente y recursos, permitió determinar capacidades. La capacidad interna, que tiene como categoría la capacidad directiva asociada a la buena comunicación o la forma de gobierno con unidades administrativas —unidad político-administrativa del resguardo-, administra y centraliza funciones legislativas — plan de vida del resguardo inga - La capacidad financiera, cuya categoría es la toma de decisiones con unidad de alto impacto que representa la toma de decisiones basadas en estados financieros, es una fortaleza que le permite al resguardo conocer su situación financiera y actuar de una manera adecuada frente a inversiones que mejoren la rentabilidad. Sin embargo, la falta de gestión ante las diferentes entidades públicas y/o privadas hace que el resguardo no tenga capital suficiente para atender sus compromisos.

La capacidad de talento humano, con la categoría fortaleza, en la cual, la comunidad Inga, con impacto medio aplica su conocimiento y prácticas ancestrales y tradicionales, adquiriendo mayor experiencia frente a otros productores. Capacidad competitiva con categoría posición de marca, se observó con ausencia de actividades de mercadeo, al no existir organización en ventas y necesidades del mercado satisfechas, con consecuencia pérdida de clientes y bajo ingreso. Las interacciones entre el estado compra de productos con variable género no es significativa ( $p$ > $0,05)$, mientras, edad afecta a $\pi 01$ como a $\pi 11$. De esta manera, $\delta t$ indica la inercia en estratos, tal que inercia en A es mayor, igual 0 menor que en $\mathrm{B}$ si $\delta_{\mathrm{t}}^{\mathrm{A}}-\delta_{\mathrm{t}}^{\mathrm{B}}>0 ;=0 ;<0$ siendo $\delta_{\mathrm{t}}^{\mathrm{A}}$ y $\delta_{\mathrm{t}}^{\mathrm{B}}$, es decir para:

$$
\begin{aligned}
& \pi_{01}(\mathrm{t})=\mathrm{P}\left(\mathrm{Y}_{\mathrm{tv}}=1 / \mathrm{Y}_{(\mathrm{t}-1)}=0\right) \text { y } \pi_{11}(\mathrm{t})=\mathrm{P}\left(\mathrm{Y}_{\mathrm{t}}=1 / \mathrm{Y}_{(\mathrm{t}-1)}=1\right) \\
& \delta_{\mathrm{t}}^{\mathrm{A}}-\delta_{\mathrm{t}}^{\mathrm{B}}=\left(\operatorname{logit} \pi_{11}^{\mathrm{A}}(\mathrm{t})-\operatorname{logit} \pi_{01}^{\mathrm{A}}(\mathrm{t})\right)-\left(\operatorname{logit} \pi_{11}^{\mathrm{B}}(\mathrm{t})-\operatorname{logit} \pi_{01}^{\mathrm{B}}(\mathrm{t})\right)
\end{aligned}
$$

Donde el modelo (1) considera estar desempleado. Se interpreta edad con efectos sobre la compra de productos, con modelos según la edad para cada periodo de transición. 
Marzo 2017 - octubre 2017: $\operatorname{logit} \mathrm{P}_{\mathrm{t}}\left(\mathrm{Y}_{\mathrm{it}}=1 / \mathrm{Y}_{\mathrm{i}(\mathrm{t}-1)}, \mathbf{X}_{\mathrm{i}(\mathrm{t}-1)}\right)$ $=\gamma_{1}-0,170 \mathrm{X}_{2}+0,0014 \mathrm{X}_{2}^{2}$

Octubre 2018 - marzo 2018: $\operatorname{logit} \mathrm{P}_{\mathrm{t}}\left(\mathrm{Y}_{\mathrm{it}}=1 / \mathrm{Y}_{\mathrm{i}(\mathrm{t}-1)}, \mathbf{X}_{\mathrm{i}(\mathrm{t}-1)}\right)$ $=\gamma_{1}-0,132 \mathrm{X}_{2}+0,0015 \mathrm{X}_{2}^{2}$ (6)

Marzo 2018 - octubre 2018: $\operatorname{logit} \mathrm{P}_{\mathrm{t}}\left(\mathrm{Y}_{\mathrm{it}}=1 / \mathrm{Y}_{\mathrm{i}(\mathrm{t}-1)}, \mathbf{X}_{\mathrm{i}(\mathrm{t}-1)}\right)$ $=\gamma_{1}-0,183 \mathrm{X}_{2}+0,0015 \mathrm{X}_{2}^{2}(7)$

En la tabla 3 se observan coeficientes diferentes de cero.
Existe entonces una influencia alta de la edad sobre la compra de productos indígenas. Las curvas indican que la probabilidad de compra disminuye en edades inferiores a 30 años, y se incrementa a partir de esta. El signo positivo indica que entre una persona que no adquiere productos con nivel afectivo estable y otro que no es estable y tiene estado emocional bajo, la probabilidad de no compra es mayor en el que posee mejor nivel afectivo.

Tabla 3. Diferencia en logit de probabilidad de pasar a no compra de producto e inercia adquisitiva de consumidores $\left(\mathrm{A}_{\mathrm{j}}\right)$ de nivel educativo inferior en comparación con los consumidores con alto nivel educativo $\left(B_{\mathrm{j}}\right)$

\begin{tabular}{|l|c|c|c|}
\hline $\begin{array}{l}\text { Nivel marzo 2018 }- \\
\text { noviembre 2018 }\end{array}$ & $\begin{array}{l}\text { Est. previo no compra } \\
\log i t \pi_{11}^{\mathrm{Aj}_{1}}(\mathrm{t})-\operatorname{logit} \pi_{11}^{\mathrm{B}}(\mathrm{t})\end{array}$ & $\begin{array}{l}\text { Est. previo compra } \\
\log i t \pi_{01}^{A j}(t)-\log i t \pi_{01}^{B}(t)\end{array}$ & $\begin{array}{l}\text { Cambio en la inercia } \\
\text { nivel educativo } \\
\delta^{A j}-\delta^{B}\end{array}$ \\
\hline Inferior a bachiller $\left(\mathbf{A}_{1}\right)$ & $-0,7703$ & $-0,1910$ & $0,993^{*}$ \\
\hline Bachiller $\left(\mathbf{A}_{2}\right)$ & $-0,2098$ & $-0,4087$ & $0,999^{*}$ \\
\hline Pregrado o maestría $\left(\mathbf{A}_{3}\right)$ & $-0,7696$ & $-0,1600$ & 0,735 \\
\hline Doctorado $\left(\mathbf{A}_{4}\right)$ & 1,984 & $-0,8950$ & $2,023 *$ \\
\hline
\end{tabular}

Fuente: elaboración propia basada en los datos obtenidos de entrevistas y encuestas (2019).

Los signos negativos en compra indican que $\pi_{01}^{\mathrm{Aj}}(\mathrm{t})<\pi_{01}^{\mathrm{B}}(\mathrm{t})$, es decir, la probabilidad de no compra es mayor en profesionales con doctorado. El signo positivo en no compra indica que la probabilidad de permanecer en no compra es menor para profesionales con doctorado que para quienes tienen pregrado, maestría o grado inferior a estos. Por otro lado, los consumidores con problemas afectivos, presentan una probabilidad alta de compra.

El análisis estructural señala que la representación cultural influye de manera significativa y de forma directa en el

\section{DISCUSIÓN}

Atendiendo las afirmaciones de Ibáñez (1994) en el modelo predictivo logit, existe similitud en el porcentaje de personas que pasan de un estado de mercadeo a otro en los periodos marzo-octubre de 2017, octubre 2017-marzo 2018 y marzo-octubre 2018. Sin embargo, se rechaza la estacionalidad de tasas de transición (Gavarrete, 2012); la heterogeneidad atribuida a la transición octubre 2017marzo 2018 ( $p$ > 005) no difiere significativamente. El modelo muestra variables asociadas a las configuradas por Leininger (1999), quien planteó que se debe garantizar asistencia, formación y atención coherentes con la cultura de cada individuo. Así también son los planteamientos de Solomon (2008), para quien los actos y las decisiones de formación se deben ajustar a los valores, la cultura, las percepciones y los estilos de vida, con el fin de potenciar los niveles cognitivos, la creatividad y la innovación. Los comportamiento del consumidor de productos en la oferta de las tribus. Los factores emocionales y espirituales asociados al afecto, los factores individuales como las creencias y las conductas aprendidas de los padres, la necesidad de sanación o el gusto por los aspectos culturales influyen en la compra. Las definiciones de la tribu y la teoría coinciden significativamente por cuanto los principios del marketing, así mismo emocionales, psicológicos, espirituales, son expresados por la tribu y los transeúntes consumidores.

resultados son similares a los hallados por Vergel, Zafra y Parra (2018) en estudios de pertinencia de programas de formación y capacitación. Con base en las afirmaciones de Bauman (2010), quien afirmó "un mundo ya no conformado por ciudadanos sino por individuos consumidores", asociadas a incertidumbre, líderes, coraje e individualidades, se puede establecer que hubo probabilidad asociada. La bondad de ajuste del modelo es buena (D'ambrosio, 2008). Tal como lo señalan Barbosa, Gómez y Leuro (2017), el lenguaje ambiental se refleja en la comunidad y en su quehacer, más allá de las creencias. La protección del medio ambiente y la creación de escenarios para la generación de medicinas y la adoración de la naturaleza son parte de su cultura. De acuerdo con Suárez, Acevedo y Huertas (2009), su lenguaje permite establecer una relación entre el lenguaje espacial y la realidad del lugar que genera el reconocimiento del espacio en otra dimensión. 
Se destaca de los resultados que la capacidad de talento humano frente al uso de prácticas ancestrales y sus factores asociados — como el social, el producto y el proceso tecnológico - afectan de manera indirecta el comportamiento del consumidor. Por otra parte, contrario a las afirmaciones de Ortiz (2016), los resultados permiten observar que el género no influye en la compra de productos. El nivel educativo muy alto predice menor adquisición de productos ofertados por las comunidades indígenas, y el estado emocional o afectivo bajo predice mayor tendencia a compra y adquisición de productos elaborados por estas.

\section{CONCLUSIÓN}

El modelo predicitivo logit $\pi_{01}(\mathrm{t})=\mathrm{P}\left(\mathrm{Y}_{\mathrm{tv}}=1 / \mathrm{Y}_{(\mathrm{t}-1)}=0\right)$; $\pi_{11}(\mathrm{t})=\mathrm{P}\left(\mathrm{Y}_{\mathrm{t}}=1 / \mathrm{Y}_{(\mathrm{t}-1)}=1\right)$ donde las variables edad, estado emocional y nivel educativo son significativas y aportan al modelo. En este modelo, el comportamiento del consumidor es influenciado por las conductas culturales utilizadas por la tribu inga para el mercadeo de sus productos, derivadas del grupo social con conocimiento en los modos de preparación, conservación y administración de medicamentos naturales. Sus secretos no se transmiten a ningún miembro que no sea de la tribu. Mediante las creencias se pueden identificar las necesidades de los consumidores, y sobre la base de esto es posible diseñar estrategias de mercadeo orientadas a un mercado que los prefiere, por ser una población vulnerable. De igual manera, los factores emocionales y espirituales asociados al afecto, así como las creencias, las conductas aprendidas de los padres, la necesidad de sanación y el gusto por los aspectos culturales influyen en las compras.

En cuanto a los elementos culturales reconocidos a través de las referencias locales, su representación no recurre a términos cartesianos para considerar las relaciones entre el individuo, el contexto sociocultural y los conocimientos y saberes que componen la cultura; al contrario, se dinamizan para plantear formas que permitan organizar y ordenar el espacio según su entorno físico, la visión y los valores del pueblo, de forma que generen sus propios términos y el lenguaje para representar.

\section{Declaración sobre conflictos de interés}

Las ideas planteadas en este artículo resultado de investigación, así como las opiniones, los comentarios y las conclusiones son responsabilidad de sus autores, y son independientes de su institución de afiliación. Así mismo, durante la realización de la investigación y la redacción de este documento no se ha incurrido en ningún conflicto de interés.

\section{REFERENCIAS BIBLIOGRÁFICAS}

Bauman, Z. (2010). Mundo consumo. Barcelona: Paidós.

https://www.planetadelibros.com/libro-

mundo-consumo/24190

Barbosa, E. M., Gómez, D. T., y Leuro, J. A. (2017). Ecología y bioeconomía. El diálogo de saberes. Clío América, 11(21), 108-119. https://doi.org/10.21676/23897848.2086

D’ambrosio, U. (2008). Etnomatemática: eslabón entre las tradiciones y la modernidad. México D.F: Limusa. http://dct.digitalcontent.com.co/sview/?i=4 $127 \& \mathrm{p}=1 \&$ idtienda=1\&token=880D3932D024-4817-B432-

FB46D126946A\& code $=4937 F 437-9545-$ 450B-BD5C-43BB1F161777

Duarte, M., Ortega, M., Mora, L. y Coromoto, N. (2011). Un modelo de atención en salud al pueblo wayuu en la frontera colombovenezolana. Revista Panamericana Salud Publica, $\quad 30(3), \quad 272-278$. https://scielosp.org/pdf/rpsp/2011.v30n3/27 2-278/es

García, D. (2012). Etnomatemática de un grupo de niños de la Granja Infantil Jesús de la Buena Esperanza de Pereira. Pereira: Universidad Tecnológica de Pereira. EtnomatematicaDeUnGrupoDeNinosDeLa GranjaInfantilJe-4271776.pdf

Gavarrete, M. H. (2012). Modelo de aplicación de etnomatemática en la formación de 
profesores para contextos indígenas en Costa Rica. España: Universidad de Granada. http://hdl.handle.net/10481/27806 Guber, R. (2011). La etnografía: método, campo y reflexividad. Buenos Aires: Siglo XXI Editores.

http://facultad.pucp.edu.pe/comunicaciones /ciudadycomunicacion/wpcontent/uploads/2014/11/Guber_Rosana__La_Etnografia_Metodo_Campo_y_Reflex ividad.pdf

Hevia, J. (2002). Lenguas y devenires en pugna. Lima: Universidad de Lima. http://www.ulima.edu.pe/publicaciones/len guas-y-devenires-en-pugna-en-torno-laposmodernidad

Jaramillo, O. (1984). Condiciones de salud y del medio ambiente de la comunidad indígena barí. Bogotá: Ministerio de Salud.

Jovchelovit, S. (2001). Social representations, public life, and social construction. En K. Deaux y G.Philogène (Eds.), Representations of the social. Bridging theoretical traditions (pp. 165-182). Hoboken, Nueva Jersey: Blackwell Publishing. http://eprints.lse.ac.uk/2649/1/Socialrepspu bliclife.pdf

Leininger, M. (1999). Cuidar a los que son de culturas diferentes requiere el conocimiento y las aptitudes de la enfermería transcultural. Cultura de los Cuidados, 3(6), 5-12. http://dx.doi.org/10.14198/cuid.1999.6.01
López, M. (2011). Estrategias de atención en salud a población indígena y su relación con la respuesta social en la ciudad de Medellín Colombia. Medellín: Universidad de Antioquia.

https://www.medellin.gov.co/irj/go/km/doc s/wpccontent/Sites/Subportal\%20de1\%20Ci udadano/Salud/Secciones/Publicaciones/Do cumentos/2012/Investigaciones\%2020112012/Estrategias\%20de\%20atención\%20en $\%$ 20salud\%20a\%20población\%20ind\%C3 \%ADgena\%202011.pdf

López, P. (2004). Población muestra y muestreo. Cochabamba, 9(8), 69-74. http://www.scielo.org.bo/scielo.php?script= sci_arttext\&pid=S1815$\underline{02762004000100012}$

Marcillo, M. y Zambrano, P. (2010). Determinantes de la participación en el mercado de trabajo. $\underline{\text { Un estudio para el área metropolitana de }}$ Pasto. Tendencias, (11), 75-96. DeterminantesDeLaParticipacionEnElMerc adoDeTrabajo-3640460.pdf

Martínez, J., Vergel, M. y Zafra, S. (2016). Comportamiento juvenil y desarrollo de competencias prosociales. Bogotá: Editorial Ibáñez.

http://incabook.com/index.php?route=prod uct/product\&product_id=6844

Murillo, J. (2010). Investigación etnográfica. México: Trillas. https://fundacionmerced.org/bibliotecadigit 
COMPORTAMIENTO DEL CONSUMIDOR DE PRODUCTOS EN LA OFERTA DE TRIBU INGA

$\mathrm{al} / \mathrm{wp}-$

content/uploads/2017/12/I_Etnografica.pdf

Ortiz, N. (2016). Tribus de consumo. Hacia la autosegmentación del consumidor. Correspondencias \& Análisis, (6), 121-137. https://doi.org/10.24265/cian.2016.n6.07

Rojas, J., Vergel, M. y Urbina, J. (2019). Imaginarios de educación superior en la juventud sorda. Bogotá: ECOE Ediciones. https://www.researchgate.net/publication/3 40297052_Imaginarios_de_educacion_supe rior_en_la_juventud_sorda

Rueda, N., Romero, D. y Gómez, C. S. (2019). Factores que inciden en la aplicación de números racionales en educación básica secundaria en una zona de frontera. Aibi: Revista de Investigación, Administración e Ingeniería, $\quad 7(2), \quad$ 16-19. https://doi.org/10.15649/2346030X.574

Sarrazin, J. P. (2015). Representaciones sobre lo indígena y su vínculo con tendencias culturales globalizadas. Anagramas, 14(27), 163-184.

RepresentacionesSobreLoIndigenaYSuVin culoConTenden-5284751.pdf

Salaverry, O. (2010). Interculturalidad en salud. Revista Perú Salud Pública, 27(1), 80-93. http://www.scielo.org.pe/scielo.php?script= sci_arttext\&pid=S172646342010000100013\&lng=es\&tlng=es.

Solomon, M. (2008). Comportamiento del consumidor. México: Pearson. https://profesorailleanasilva.files.wordpress .com/2016/06/comportamiento-delconsumidor-michael-solomon.pdf

Spradley, J. (2008). The ethnographic interview. Winston: Universidad de Michigan. https://books.google.com.co/books/about/T he_Ethnographic_Interview.html?id=XP5_ AAAAMAAJ\&redir_esc=y

Suárez, I., Acevedo, M. y Huertas, C. (2009). Etnomatemática, educación matemática e invidencia. Revista Latinoamericana de Etnomatemática, $\quad 2(2), \quad$ 18-51. http://www.etnomatematica.org/v2-n2agosto2009/suarez-acevedo-huertas.pdf

Vergel-Ortega, M., Delgado, J. y Díaz-Umaña, Y. (2019). Cathedral bell's San José de Cúcuta: Heritage and acoustics. Journal of Physics: Conference Series, 1329(1), 1-7. 10.1088/1742-6596/1329/1/012004

Vergel, M., Martínez, J. y Nieto, J. F. (2016). $\underline{\text { Validez de instrumento para medir el }}$ aprendizaje creativo. Revista Comunicaciones en Estadística, 9(2), 239254. https://doi.org/10.15332/s2027$\underline{3355.2016 .0002 .04}$

Vergel, M., Zafra, S. L. y Parra, H. M. (2018). Modelo para evaluar la pertinencia de programas en educación superior. Caso: Universidad Francisco de Paula Santander. Alemania: Editorial Académica Española. https://www.morebooks.de/store/es/book/m odelo-para-evaluar-la-pertinencia-deprogramas-en-educación-superior/isbn/978620-2-13104-9 
Mawency Vergel-Ortega, Luisa Stella Paz-Montes y Jhan Piero Rojas-Suárez

Vilela, G., Vergel, M. y Paz L. S. (2018). Social Ciencia y Tecnologia, 10(4), 64-73. representations in the interaction with http://dx.doi.org/10.22335/rlct.v10i4.664 students of the Inga tribes. Revista Logos 